\title{
Quantum Shuttle in Phase Space
}

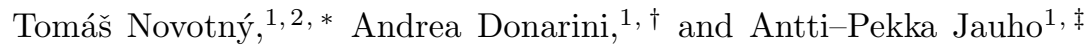 \\ ${ }^{1}$ Mikroelektronik Centret, Technical University of Denmark, \\ Ørsteds Plads, Bldg. 345 east, DK-2800 Kgs. Lyngby, Denmark \\ ${ }^{2}$ Department of Electronic Structures, Faculty of Mathematics and Physics, \\ Charles University, Ke Karlovu 5, 12116 Prague, Czech Republic
}

(Dated: November 17, 2018)

\begin{abstract}
We present a quantum theory of the shuttle instability in electronic transport through a nanostructure with a mechanical degree of freedom. A phase space formulation in terms of the Wigner function allows us to identify a cross-over from the tunnelling to the shuttling regime, thus extending the previously found classical results to the quantum domain. Further, a new dynamical regime is discovered, where the shuttling is driven exclusively by the quantum noise.

PACS numbers: 05.60.Gg, 73.23.Hk, 85.85.+j
\end{abstract}

Advances in microfabrication technology are pushing today's microelectromechanical systems (MEMS) towards the nanometer regime, and the emerging new technology of nanoelectromechanical systems (NEMS) is expected to play an important role in the future. The ubiquitous quantum mechanical effects affecting the performance of these devices present many theoretical challenges, only few of which have so far been addressed in the literature. The purpose of this Letter is to present a fully quantum theory for an electromechanical instability in a generic NEMS device, the single-electron shuttle first studied by Gorelik et al [1] .

This device consists of a movable single-electron transistor (SET) and exhibits an electromechanical instability from the standard tunnelling regime to a new regime in which the SET oscillates and carries an integer number of electrons per a cycle (shuttle regime). Since the original suggestion [1], there has been increasing interest in the shuttle phenomenon [2, 3, 4, 5, 6, 7, $8,29,10]$ : e.g. by incorporating the shot noise due to the electron transfer [2], gate effects 3], the coherence effects in the electronic subsystem [4] and strong dissipation of the oscillator energy [5]. Only very recently the quantum mechanical treatment of the oscillations in various modifications of the shuttle setup has been considered [ $6,[8]$.

The classical theory of shuttle transport has been used [3, 4] to describe the experiments on $\mathrm{C}_{60}$ single-electron transistor 11], where the oscillations of the center of mass of the molecule were found to be important. However, also explanations based on incoherent phonon assisted tunnelling theory which do not take into account the correlation between the coherent oscillator motion and the electron transfer seem to yield reasonable predictions for the I-V curves 12, 13].

Therefore, a fully coherent quantum mechanical treatment of the oscillator which does take into account possible correlations and which would, in principle, allow to join the two approaches into a unified framework is a most desirable theoretical task. In this study we present an attempt for such a treatment for a simplest model supporting the shuttling transition in the classical regime. A complementary study to ours already exists [10] which uses a similar model, albeit without any mechanical damping. However, the quasiclassical expansion of the tunneling term used in that work does not give access to the purely quantum phenomena discussed below.

We demonstrate that the characteristic strong correlation between the oscillator motion and the electron transfer persists even in the quantum regime. The noise generated by various sources (shot and thermal, both having quantum components) is found to be very important for the phenomenon. Not only does it smear the classical transition found in [1] into a crossover with a considerably shifted position in parameter space compared to the classical "mean field" study [1, 4] but the quantum component of the shot noise also generates the shuttle instability even in the classically stable region with zero electric field.

Using the generalized master equation approach suggested in [6] we study a simple model motivated by several previous studies [1, 4, 10, 13]. Namely, we consider an oscillating nanoscopic grain with only one electronic level (strict Coulomb blockade regime) coupled to two leads. The oscillator degree of freedom is treated fully quantum mechanically in our approach. We also account for the oscillator damping, the thermal noise due to the oscillator-bath coupling, and the shot noise due to the quantized electron transfer.

The Hamiltonian of the model reads

$$
\begin{array}{r}
H=H_{\mathrm{osc}}+\epsilon_{0} c_{0}^{\dagger} c_{0}+\sum_{k ; \alpha=L, R}\left(\epsilon_{k \alpha}-\mu_{\alpha}\right) c_{k \alpha}^{\dagger} c_{k \alpha}+H_{B} \\
-e E x c_{0}^{\dagger} c_{0}+\sum_{k ; \alpha=L, R}\left(T_{k \alpha 0}(x) c_{k \alpha}^{\dagger} c_{0}+\text { h.c. }\right)+H_{\mathrm{int}}
\end{array}
$$

where the first three terms describe the free evolution of a linear harmonic oscillator $H_{\text {osc }}=\frac{p^{2}}{2 m}+\frac{m \omega^{2} x^{2}}{2}$, the single electronic level on the grain, and the two noninteracting leads symmetrically biased by voltage $-V$ (i.e. 
$\mu_{L}=\frac{e V}{2}, \mu_{R}=-\frac{e V}{2}$ with $e, V>0$; all electronic energies are measured with respect to the equilibrium chemical potential of the leads), respectively. The term $H_{B}$ describes a generic Ohmic heat bath 14. The first interaction term accounts for the electrostatic coupling between the position of the grain and the occupation of the grain electronic level; $E$ is the electric field caused by the bias applied between the leads and/or gate voltage $[3,13]$. This electric field causes a shift of the charged oscillator equilibrium by $d=\frac{e E}{m \omega^{2}}$. The second term is the coupling of the leads to the single electronic state on the grain with oscillator-dependent hopping amplitudes $T_{k L 0}(x)=t_{L} \exp \left(-\frac{x}{\lambda}\right), T_{k R 0}(x)=t_{R} \exp \left(\frac{x}{\lambda}\right)$ where $\lambda$ is the electron tunnelling length. The last term $H_{\text {int }}$ describes the coupling of the heat bath to the oscillator which is linear in the oscillator coordinate 14. The net effect of this coupling is the oscillator mechanical damping characterized by a constant $\gamma$ and the Langevin random force that the bath exerts on the oscillator and which depends on the temperature of the bath $T$.

By projecting out the leads and the thermal bath we arrive at a Markovian generalized master equation (GME) for the density matrix $\rho(t)$ of the system composed of the grain and the harmonic oscillator. Since the electronic transfer rate in the shuttling regime is comparable with the frequency of the oscillations we employed the singular coupling limit 15, 16] appropriate for rapidly decaying systems. This approximation is valid for bias much larger than any energy scale of the system, i.e. $\mathrm{eV} \gg \hbar \omega, \epsilon_{0}$. Moreover, we also assume $\mathrm{eV} \gg k_{B} T$ which is reasonable in the present physical context. The mechanical damping due to the heat bath is on the other hand treated within the standard weak coupling theory 17.

Our GME reads

$$
\dot{\rho}(t)=\mathcal{L} \rho(t)=\left(\mathcal{L}_{\text {coh }}+\mathcal{L}_{\text {driv }}+\mathcal{L}_{\text {damp }}\right) \rho(t)
$$

with

$$
\begin{aligned}
\mathcal{L}_{\mathrm{coh}} \rho & =\frac{1}{i \hbar}\left[H_{\mathrm{osc}}+\epsilon_{0} c_{0}^{\dagger} c_{0}-e \operatorname{Ex} c_{0}^{\dagger} c_{0}, \rho\right] \\
\mathcal{L}_{\text {driv }} \rho & =-\frac{\Gamma_{L}}{2}\left(c_{0} c_{0}^{\dagger} e^{-\frac{2 x}{\lambda}} \rho-2 c_{0}^{\dagger} e^{-\frac{x}{\lambda}} \rho e^{-\frac{x}{\lambda}} c_{0}+\rho e^{-\frac{2 x}{\lambda}} c_{0} c_{0}^{\dagger}\right) \\
& -\frac{\Gamma_{R}}{2}\left(c_{0}^{\dagger} c_{0} e^{\frac{2 x}{\lambda}} \rho-2 c_{0} e^{\frac{x}{\lambda}} \rho e^{\frac{x}{\lambda}} c_{0}^{\dagger}+\rho e^{\frac{2 x}{\lambda}} c_{0}^{\dagger} c_{0}\right)
\end{aligned}
$$$$
\mathcal{L}_{\text {damp }} \rho=-\frac{i \gamma}{2 \hbar}[x,\{p, \rho\}]-\frac{\gamma m \omega}{\hbar}(\bar{N}+1 / 2)[x,[x, \rho]] .
$$

Here, [,] and $\{$,$\} denote the commutator and the anti-$ commutator, respectively and $\bar{N}=\left(\exp \left(\frac{\hbar \omega}{k_{B} T}\right)-1\right)^{-1}$ is the mean oscillator occupation at the bath temperature.

The first term in Eq. (2) is the free coherent evolution of the oscillator and the grain level while the second one describes the transfer of electrons via the oscillator-position-dependent tunnel junctions from the left reservoir to the grain level (the term with $\Gamma_{L}$ ) and from the level to the right reservoir (the term with $\Gamma_{R}$ ). The opposite processes can be neglected due to the assumption $e V \gg \hbar \omega, \epsilon_{0}, k_{B} T$. The transfer rates equal $\Gamma_{L, R}=\frac{2 \pi}{\hbar}\left|t_{L, R}\right|^{2} \mathcal{D}_{L, R}$ with the constant densities of states of the leads $\mathcal{D}_{L, R}$. The third term accounts for the interaction of the oscillator with the heat bath. Translational invariance of the damping, positivity of the density matrix, and relaxation towards canonical equilibrium cannot be achieved simultaneously with any Markovian damping kernel and one has to sacrifice at least one of these properties. The most physical choice is to relax the positivity [17]. We checked the magnitude of breaking of the positivity in our calculations and found it irrelevant. Moreover, it only occurs for large values of $\gamma$ out of the shuttling regime.

It can be shown that the electronic off-diagonal elements of the density matrix are decoupled from the diagonal ones and, moreover, decay to zero in the stationary state. Therefore, it is sufficient to consider only the electronic diagonal elements: $\rho_{00}(t)=\langle 0|\rho(t)| 0\rangle$ and $\rho_{11}(t)=\langle 1|\rho(t)| 1\rangle$, where $|1\rangle=c_{0}^{\dagger}|0\rangle$. These objects are still full density matrices in the oscillator space and satisfy

$$
\begin{aligned}
\dot{\rho}_{00}(t) & =\frac{1}{i \hbar}\left[H_{\mathrm{osc}}, \rho_{00}(t)\right]-\frac{\Gamma_{L}}{2}\left(e^{-\frac{2 x}{\lambda}} \rho_{00}(t)+\rho_{00}(t) e^{-\frac{2 x}{\lambda}}\right) \\
& +\Gamma_{R} e^{\frac{x}{\lambda}} \rho_{11}(t) e^{\frac{x}{\lambda}}+\mathcal{L}_{\mathrm{damp}} \rho_{00}(t), \\
\dot{\rho}_{11}(t) & =\frac{1}{i \hbar}\left[H_{\mathrm{osc}}-e E x, \rho_{11}(t)\right]+\Gamma_{L} e^{-\frac{x}{\lambda}} \rho_{00}(t) e^{-\frac{x}{\lambda}} \\
& -\frac{\Gamma_{R}}{2}\left(e^{\frac{2 x}{\lambda}} \rho_{11}(t)+\rho_{11}(t) e^{\frac{2 x}{\lambda}}\right)+\mathcal{L}_{\mathrm{damp}} \rho_{11}(t) .
\end{aligned}
$$

From the continuity equation for the electronic charge we may deduce the following formula for the stationary current through the grain (flowing from the left to the right lead):

$$
I^{\text {stat }}=e \Gamma_{L} \operatorname{Tr}_{\mathrm{osc}}\left(e^{-\frac{2 x}{\lambda}} \rho_{00}^{\text {stat }}\right)=e \Gamma_{R} \operatorname{Tr}_{\mathrm{osc}}\left(e^{\frac{2 x}{\lambda}} \rho_{11}^{\text {stat }}\right) .
$$

The trace is carried out over the oscillator basis and $\rho_{n n}^{\text {stat }}=\lim _{t \rightarrow \infty} \rho_{n n}(t)$.

We solved numerically the stationary version of the above equations (6): $\quad 0=\sum_{l=1}^{2 N^{2}} L_{k l} \rho_{l}^{\text {stat }}$ where the column vector $\rho_{l}^{\text {stat }}$ consists of the matrix elements of $\rho_{00}^{\text {stat }}, \rho_{11}^{\text {stat }}$ and the (super-)matrix $L_{k l}$ of the dimension $2 N^{2} \times 2 N^{2}$ contains the appropriate coefficients of the linear system (6). The density matrix was represented in the harmonic oscillator basis which was truncated by taking up to $N=100$ lowest states which yields satisfactory numerical convergence in our parameters range. We determined the unique (for $\Gamma_{R}=\Gamma_{L}=\Gamma \neq 0$ ) null vector of the supermatrix $L$ by using the Arnoldi iteration [18]. 


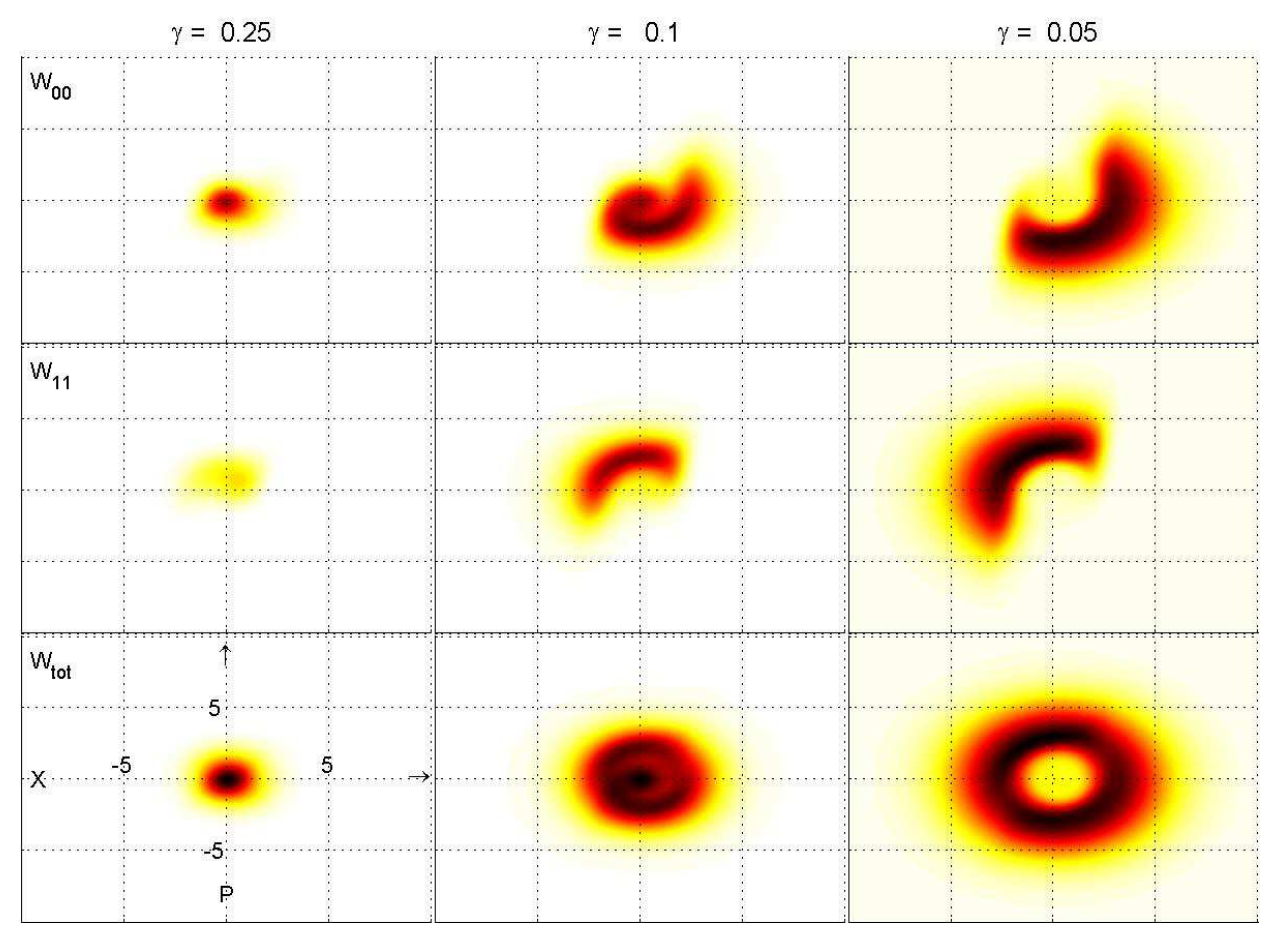

FIG. 1: Phase space picture of the tunnelling-to-shuttling crossover. The respective rows show the Wigner distribution functions for the discharged $\left(W_{00}\right)$, charged $\left(W_{11}\right)$, and both $\left(W_{\text {tot }}\right)$ states of the oscillator in the phase space (horizontal axis - coordinate in units of $x_{0}=\sqrt{\hbar / m \omega}$, vertical axis - momentum in $\left.\hbar / x_{0}\right)$. The values of the parameters are: $\lambda=x_{0}, T=0, d=0.5 x_{0}, \Gamma=$ $0.05 \hbar \omega$. The values of $\gamma$ are in units of $\hbar \omega$. The Wigner functions are normalized within each column.

Our approach differs from the one used in $[\underline{6}]$ where the explicit time integration scheme of the time-dependent equation analogous to (6) was used to determine the stationary density matrix. We applied our method to the model of [6] and recovered the I-V curve results reported there. Using the phase space analysis detailed below we fully confirmed the shuttling interpretation based on the indirect evidence from changes of the I-V curves with changing parameters.

The I-V curve (or other dependencies of the current on some parameter) alone yields only an indirect evidence of shuttling and may actually not be decisive whether the system is shuttling or not (see e.g. 12] versus [4]). Therefore, it is preferable to consider quantities which depend also on the state of the oscillator. An excellent visualization tool 17] for the description of the joint electronic and oscillator properties are the Wigner functions $(n=0,1)$

$W_{n n}(X, P)=\int_{-\infty}^{\infty} \frac{d y}{2 \pi \hbar}\left\langle X-\frac{y}{2}\left|\rho_{n n}^{\text {stat }}\right| X+\frac{y}{2}\right\rangle \exp \left(i \frac{P y}{\hbar}\right)$

yielding the charge-resolved quasiprobability distributions of the oscillator in the phase space. These functions provide us with a clear evidence of the transition from the incoherent tunnelling to the coherent quasiclassical shuttling behavior with decreasing damping coefficient.
Our focus is in the quantum effects on the shuttling transition and, in particular, whether there is any transition in the quantum regime at all. Therefore, we work in the strictly quantum regime where the tunnelling length is comparable to the zero uncertainty of the oscillator, $\lambda \sim x_{0}=\sqrt{\hbar / m \omega}$, and where only a relatively small number of oscillator states is excited.

In Fig. 1 we depict the Wigner functions $W_{00}, W_{11}$, and $W_{\text {tot }}=W_{00}+W_{11}$ showing the crossover from the tunnelling to the shuttling regime with decreasing damping. In the tunnelling regime (large $\gamma$ ) the oscillator is located around the origin (or shifted origin when charged) with no particular correlation between its charge state and momentum (Wigner functions are centered around the origin with some "quantum fuzziness"). This is consistent with the quantum incoherent tunnelling picture. On the other hand, in the shuttling regime (small $\gamma$ ) the oscillator orbits almost classically (ring-like shape of $W_{\text {tot }}$ with a hole around the origin), and shuttles the charge on its way from the left to the right lead and returns empty back (half-moon shapes of $W_{00}, W_{11}$ ). The correlation between the charge state and the mechanical motion is very strong. In the crossover region (medium $\gamma$ ) we can see that both regimes of transport are contributing additively (ring-like shape plus an incoherent peak around the origin of $\left.W_{\text {tot }}\right)$. The classical "mean field" 


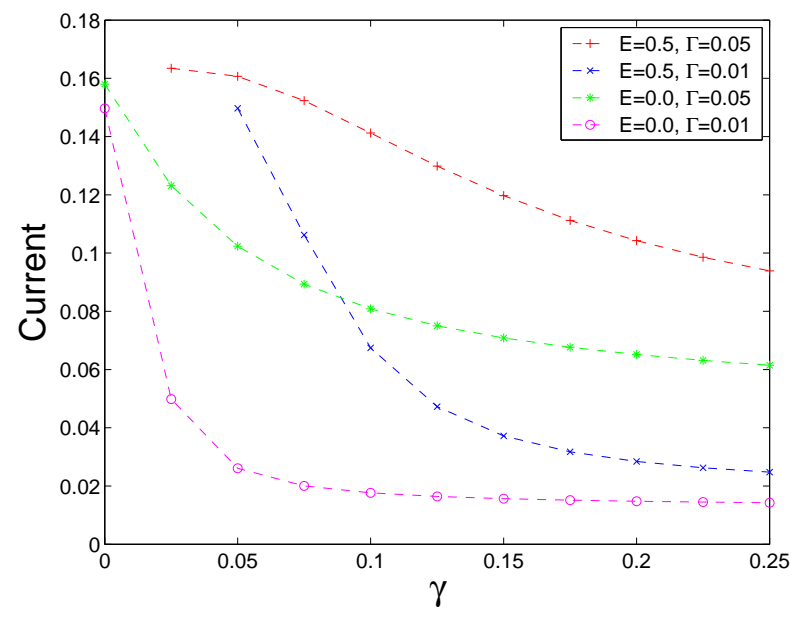

FIG. 2: $I-\gamma$ curve. The $\gamma$-dependence of the stationary current through the grain for different transfer rates and electric fields. Their values are $d=0.5 x_{0}, \Gamma=0.05 \hbar \omega$ (pluses; corresponds to Fig. 1), $d=0.5 x_{0}, \Gamma=0.01 \hbar \omega$ (circles), $d=0.0, \Gamma=0.05 \hbar \omega$ (asterisks), $d=0.0, \Gamma=0.01 \hbar$ (crosses). Other parameters are $\lambda=x_{0}, T=0$. The current is in units of $e \omega$ while $\gamma$ in $\hbar \omega$.

sharp transition of 1$]$ between the tunnelling and the shuttling regime is smeared into a crossover due to the noise. Also the position of the crossover is substantially shifted with respect to the classical values $\left(\gamma_{\text {cross }}\right.$ is $\sim 5$ times larger than the classical value) which is attributed to the deeply quantum (and therefore noisy) regime. The classical picture is expected to emerge in the quasiclassical limit $d, \lambda \gg x_{0}$.

In Fig. 2 we plot the $\gamma$-dependence of the stationary current through the grain for different transfer rates $\Gamma$ and electric fields (measured in $d$ ). For the two curves with $d \neq 0$ we can see the rise of the current in the crossover region from the tunnelling-limited values proportional to $\Gamma$ to shuttling-mediated quantized value of one shuttled electron per each cycle $(1 / 2 \pi \approx 0.16$ in our units; independent of $\Gamma$ ) in agreement with the classical results. More surprisingly, also the results for the case of zero electric field $d=0$ show clear signs of shuttling crossover in the $I-\gamma$ curve for small enough mechanical damping. Classically there is no shuttling transition for $d=0$ regardless of the other parameters values [4] and the $I-\gamma$ curve is constant. Therefore, this shuttling must be driven purely by the quantum component of the shot noise (proportional to $\hbar$ ). Also the phase space pictures of this regime reveal onset of shuttling transport.

Finally, we comment on the effect of the temperature. For a nonzero temperature the shuttling transition within our model is facilitated by the increase of the mechanical noise driving the transition. The deteriorating effect of the temperature on the transition is not included in the model due to the high bias assumption. The development of the theory for a finite bias is under way.

To summarize, we have presented a quantum theory of the shuttling transition in fully developed Coulomb blockade regime. Using the Wigner functions as a phase space visualization method we have exhibited a clear crossover from the tunnelling to the shuttling regime of the transport as a function of the mechanical damping parameter. The effect of noise on the transition in the deeply quantum regime $\left(\lambda \sim x_{0}=\sqrt{\hbar / m \omega}\right)$ is pronounced and can even trigger the transition in the classically stable regime with the zero electric field.

The authors want to thank the members of the Chalmers group, A. Wacker, and B. Velický for helpful discussions. Advice from T. Eirola concerning numerical methods was indispensable. Support of the grant 202/01/D099 of the Czech grant agency for one of us (T.N.) is also gratefully acknowledged.

* Electronic address: tno@mic.dtu.dk

$\dagger$ Electronic address: ad@mic.dtu.dk

‡ Electronic address: antti@mic.dtu.dk

[1] L. Y. Gorelik, A. Isacsson, M. V. Voinova, B. Kasemo, R. I. Shekhter, and M. Jonson, Phys. Rev. Lett. 80, 4526 (1998), cond-mat/9711196.

[2] A. Isacsson, L. Y. Gorelik, M. V. Voinova, B. Kasemo, R. I. Shekhter, and M. Jonson, Physica B 255, 150 (1998), cond-mat/9804281.

[3] N. Nishiguchi, Phys. Rev. B 65, 035403 (2001).

[4] D. Fedorets, L. Y. Gorelik, R. I. Shekhter, and M. Jonson, Europhys. Lett. 58, 99 (2002), cond-mat/0104200.

[5] T. Nord, L. Y. Gorelik, R. I. Shekhter, and M. Jonson, Phys. Rev. B 65, 165312 (2002), cond-mat/0106589.

[6] A. D. Armour and A. MacKinnon, Phys. Rev. B 66, 035333 (2002), cond-mat/0204521.

[7] N. Nishiguchi, Phys. Rev. Lett. 89, 066802 (2002).

[8] A. Y. Smirnov, L. G. Mourokh, and N. J. M. Horing, Phys. Rev. B 67, 115312 (2002), cond-mat/0209149.

[9] L. Y. Gorelik, A. Isacsson, Y. M. Galperin, R. I. Shekhter, and M. Jonson, Nature 411, 454 (2001).

[10] D. Fedorets, L. Y. Gorelik, R. I. Shekhter, and M. Jonson, Quantum precursor of shuttle instability (2002), condmat/0212561.

[11] H. Park, J. Park, A. K. L. Lim, E. H. Anderson, A. P. Alivisatos, and P. L. McEuen, Nature 407, 57 (2000).

[12] D. Boese and H. Schoeller, Europhys. Lett. 54, 668 (2001), cond-mat/0012140.

[13] K. D. McCarthy, N. Prokof'ev, and M. T. Tuominen, Incoherent dynamics of vibrating single-molecule transistors (2002), cond-mat/0205419.

[14] U. Weiss, Quantum Dissipative Systems, vol. 10 of Series in Modern Condensed Matter Physics (World Scientific, 1999), 2nd ed.

[15] H. Spohn, Rev. Mod. Phys. 52, 569 (1980).

[16] G. Kimura, K. Yuasa, and K. Imafuku, Phys. Rev. A 63, 22103 (2001).

[17] D. Kohen, C. C. Marston, and D. J. Tannor, J. Chem. Phys. 107, 5236 (1997).

[18] G. H. Golub and C. F. V. Loan, Matrix Computations (The John Hopkins University Press, 1996), 3rd ed. 